\title{
Potential factors involved in virulence of Cronobacter sakazakii isolates by comparative transcriptome analysis
}

\author{
Yingwang Ye, ${ }^{*} \dagger^{1}$ Xiyan Zhang, ${ }^{*}$ Maofeng Zhang, ${ }^{*}$ Na Ling, $\dagger$ Haiyan Zeng, $\dagger$ Jina Gao, ${ }^{*}$ Rui Jiao, ${ }^{*}$ \\ Qingping Wu, $†$ and Jumei Zhang† \\ *School of Food Science and Technology, Hefei University of Technology, Hefei, 230009, China \\ †State Key Laboratory of Applied Microbiology, South China (the Ministry-Province Joint Development), Provincial Key Laboratory \\ of Microbiology Culture Collection and Application, Guangdong Institute of Microbiology, Guangzhou, 510070, China
}

\section{ABSTRACT}

Cronobacter species are important foodborne pathogens causing severe infections in neonates through consumption of contaminated powdered infant formula. However, the virulence-associated factors in Cronobacter are largely unknown. In this study, the transcriptome analysis between highly virulent Cronobacter sakazakii G362 and attenuated L3101 strains was used to reveal the potential factors involved in virulence. The total transcripts were grouped into 20 clusters of orthologous group categories and summarized in 3 gene ontology categories (biological process, cellular component, and molecular function). In addition, the differentially expressed genes (DEG) between these isolates were analyzed using Volcano plots and gene ontology enrichment. The predominant DEG were flagellaassociated genes such as $f l h D, \operatorname{mot} A, f \lg M, f l g B$, and fliC. Furthermore, the expression abundance of outer membrane protein or lipoprotein genes ( omp $W$, slyB, $b l c$, tolC, and lolA), potential virulence-related factors (hlyIII and hha), and regulation factors (sdiA, che $Y$, $B s s, f l i Z)$ was also significantly different between G362 and L3101. Interestingly, 3 hypothetical protein genes (ESA_01022, ESA_01609, and ESA_00609) were found to be expressed only in G362. Our findings provide valuable transcriptomic information about potential virulence factor genes, which will be needed in future molecular biology studies designed to understand the pathogenic mechanism of Cronobacter.

Key words: Cronobacter, transcriptome analysis, virulence factor, flagella, outer membrane protein

Received February 25, 2017.

Accepted July 11, 2017.

${ }^{1}$ Corresponding author: yeyw04@mails.gucas.ac.cn

\section{INTRODUCTION}

Cronobacter spp. (formerly known as Enterobacter sakazakii) are foodborne pathogens associated with severe infections in neonates through consumption of contaminated powdered infant formula $(\mathrm{FAO} / \mathrm{WHO}$, 2008; CDC, 2012), and hazards of Cronobacter species in dairy products are of great concerns. Recently, it has been reported that the genus of Cronobacter consisted of 7 species: Cronobacter sakazakii, Cronobacter malonaticus, Cronobacter turicensis, Cronobacter muytjensii, Cronobacter dublinensis, Cronobacter condimenti, and Cronobacter universalis (Iversen et al., 2008; Joseph et al., 2012), and genomic analysis showed that only strains from C. sakazakii, C. malonaticus, and C. turicensis were associated with severe infections (Kucerova et al., 2010).

Pagotto et al. (2003) first described the virulence differences among Cronobacter isolates. In addition, essential roles of outer membrane proteins (ompA and ompX) were observed in invasion and adhesion to Caco-2 and INT-407 (Mohan Nair and Venkitanarayanan, 2007; Mittal et al., 2009; Kim et al., 2010). The cell-bound zinc-containing metalloprotease encoded by the $z p x$ gene was considered to be the potential virulence factors (Kothary et al., 2007). Putative virulence genes on the related RepFIB plasmids harbored by Cronobacter species were characterized by Franco et al. (2011). The LysR-type transcriptional regulator homolog was found to play an important role in the virulence of C. sakazakii ATCC29544 (Choi et al., 2012). Emami et al. (2012) reported that, compared with wild-type mice infected by C. sakazakii, depletion of PMN and macrophages in newborn mice caused increased recruitment of dendritic cells in the intestine. Our groups determined membrane protein expression differences for enzV, ompX, lptE, pstB, and OsmY between G362 and L3101, which were potentially involved in virulence (Ye et al., 2015). Ye et al. (2016) further reported that the potential proteins (Dps, ompA, and LuxS) might be related to virulence 
of C. sakazakii strains. However, global information about the molecular basis of virulence in Cronobacter is currently not available at the transcriptome level.

The RNA-seq technology has been proven to be a powerful tool for transcriptome analysis (Dugar et al., 2013). This direct sequencing methodology enables absolute gene expression information at the mRNA level. In addition, this method is more sensitive for detecting low-abundant transcripts and small changes in gene expression under different conditions ('t Hoen et al., 2008; Wilhelm et al., 2008). The RNA-seq technology has been recently applied to determine comparative transcriptome analysis in Campylobacter jejuni (Chaudhuri et al., 2011) and Salmonella enterica serovar Typhi (Perkins et al., 2009). The responses of C. sakazakii to garlic-derived organosulfur compounds were determined using high-throughput whole-transcriptome sequencing and confocal micro-raman spectroscopy (Feng et al., 2014).

In this study, Illumina mRNA sequencing (RNA-seq) was used for transcriptome analysis between highly virulent Cronobacter sakazakii G362 and attenuated L3101 strains. Our findings will contribute to our understanding about its pathogenicity and valuable information for revealing aspects of virulence in C. sakazakii.

\section{MATERIALS AND METHODS}

\section{C. sakazakii Isolates Used in This Study}

Cronobacter sakazakii G362 and L3101 strains were identified using the International Organization for Standardization method, API-20E, chromogenic media, and PCR targeting rpoB (Stoop et al., 2009) and sequencing of fusA (Baldwin et al., 2009). The virulence of Cronobacter strains G362 (virulent isolate) and L3101 (attenuated isolate) were determined through intraperitoneal injection of mice followed by histopathologic analysis of specific organs, which was previously reported by Ye et al. (2016), and the 2 isolates were further used for transcriptome analysis.

\section{RNA Extraction of G362 and L3101}

Cronobacter sakazakii G362 and L3101 strains were inoculated into tryptic soy broth (TSB, Huankai, Guangzhou, China) for $16 \mathrm{~h}$ at $37^{\circ} \mathrm{C}$. The total RNA was extracted from $1.0 \mathrm{~mL}$ of TSB enrichment culture with RNA isolation kit (Qiagen, Hilden, Germany) according to the manufacturer's instructions. Extraction of RNA was performed in triplicate for each isolate. Then, genomic DNA and rRNA of the RNA samples were depleted using DNase I (Invitrogen, Carlsbad,
CA) and a Ribominus Transcriptome Isolation Kit (Invitrogen) according to the manufacturer's instructions.

\section{cDNA Library Preparation for RNA-seq}

Construction of cDNA libraries was performed using Truseq RNA sample preparation kit according to the manufacturer's instructions (Illumina, San Diego, CA) as previously described by Dong et al. (2013). Then, the final purified DNA was captured on an Illumina flow cell for cluster generation and cDNA libraries were sequenced on the Illumina sequencing platform HiSeq 2000.

\section{Data Processing and Analysis}

The raw data were filtered to remove "dirty" reads or low-quality reads. The clean reads obtained using RNA-seq were compiled using bowtie2-2.1.0 (Slashdot Media, La Jolla, CA) read aligner software. Then, the reads were aligned to complete genome of $C$. sakazakii ATCC BAA-894. The number of each read was normalized to reads per kilobase of exon model per million mapped reads (RPKM), and thus the RPKM values were considered as the final expression levels for each gene between C. sakazakii G362 and L3101. Thereafter, all transcripts were subject to analysis of clusters of orthologous groups (COG), Kyoto Encyclopedia of Genes and Genomes (KEGG), and gene ontology (GO).

\section{Analysis of Differential Expression Genes}

A statistical analysis of the frequency of each read was performed to compare gene expression differences between G362 and L3101. According to the statistical framework, the $P$-values of differential expression significance of each transcript between G362 and L3101 were calculated according to the AC statistical framework (Audic and Claverie, 1997; Xu et al., 2013). Then, $P$-values less than 0.05 were regarded as the threshold to judge the significant differences in gene expression between strains at the mRNA level.

\section{Quantitative Analysis of Partial Differential Expression Genes Using Real-Time PCR}

For real-time PCR, primers for fliC, omp $W$, sdiA, flg $M$, and $f l h D$ genes are listed in Table 1, and the $16 \mathrm{~S}$ rRNA gene was used as the internal control. Two strains were inoculated into TSB for overnight at $37^{\circ} \mathrm{C}$, and total RNA was extracted from $1.0 \mathrm{~mL}$ of culture using a bacterial RNA extraction kit (Biomiga, San Di- 
Table 1. Primers used for real-time PCR in this study

\begin{tabular}{|c|c|c|}
\hline Gene & Primers $^{1}\left(5^{\prime}-3^{\prime}\right)$ & Size (bp) \\
\hline $16 \mathrm{~S}$ rRNA & $\begin{array}{l}\text { F: AACCCTTATCCTTTGTTGCCA } \\
\text { R: CGGACTACGACGCACTTTATG }\end{array}$ & 195 \\
\hline omp $W$ & $\begin{array}{l}\text { F: AGCGTTGACGATTCCTGGG } \\
\text { R: AGCCCGCCGAGAACATAA }\end{array}$ & 187 \\
\hline $\operatorname{flg} M$ & $\begin{array}{l}\text { F: CGAAAACAGTGAACCGCAGG } \\
\text { R: GTCCATTTTCAGCTCGCCA }\end{array}$ & 181 \\
\hline $\operatorname{flh} D$ & $\begin{array}{l}\text { F: GACTCTGCCGCAAATGGTG } \\
\text { R: CCTTTTCTTCCTGGCGACG }\end{array}$ & 199 \\
\hline sdiA & $\begin{array}{l}\text { F: AAAACTGACGCTGCACACCA } \\
\text { R: TTACGCCCTTGACCAGACC }\end{array}$ & 197 \\
\hline$f l i C$ & $\begin{array}{l}\text { F: AAACGACGGCATCTCCCTG } \\
\text { R: GCGTTGAAGTTAGCACCACCAG }\end{array}$ & 198 \\
\hline
\end{tabular}

${ }^{1} \mathrm{~F}=$ forward; $\mathrm{R}=$ reverse.

ego, CA) according to the manufacturer's instructions. Then, cDNA was obtained using a first-strand cDNA synthesis kit (Biomiga) according to the manufacturer's instructions. All samples were detected as described by Ye et al. (2015). The relative expression of different genes between G362 and L3101 was calculated using the $2^{-\Delta \Delta \mathrm{Ct}}$ method $(\mathrm{Ct}=$ cycle threshold $)$.

\section{Transmission Electron Microscope Observation of Flagella Synthesis}

After incubation in the TSB at $37^{\circ} \mathrm{C}$ for $16 \mathrm{~h}$, the flagella synthesis in isolates G362 and L3101 were negatively stained and analyzed using a transmission electron microscope (Hitachi 7650, Hitachi, Tokyo, Japan).

\section{RESULTS}

\section{IIlumina Transcriptome Sequencing and Reads Assembly}

The total transcripts are aligned with data based on NR, SWISS-PROT, TREMBL, CDD, PRAM, and COG showing percentages of 54.04, 39.86, 67.58, 37.00, 29.11 , and $16.70 \%$ annotated information, respectively, shown in Supplemental Table S1 (https://doi.org/10 $.3168 / \mathrm{jds} .2017-12801)$. In addition, most of the highest homology (72.00\%) with C. sakazakii ATCC BAA-894 was observed, followed by C. sakazakii ES15 and C. sakazakii E899 showing 22.00 and $6.00 \%$ homology based on alignment with NR data shown in Supplemental Figure S1 (https://doi.org/10.3168/jds.2017 -12801).

\section{COG and GO Classification}

To evaluate the completeness of our transcriptome library and the effectiveness of the annotation process, genes were annotated into 20 COG functional cat- egories, of which AA transport and metabolism represented the largest group $(109,24.76 \%)$, followed by carbohydrate transport and metabolism $(92,20.87 \%)$ and general functions $(83,18.83 \%)$ shown in Figure 1.

Additionally, GO functional annotations were obtained based on the BLAST uniprot in Figure 2 and were categorized into 49 functional groups. The GO terms were most represented in 3 main categories (molecular function, cellular component, and biological process).

To further survey the biological pathways that are active in C. sakazakii, the total gene sequences were subject to KEGG analysis. In Supplemental Table S2 (https://doi.org/10.3168/jds.2017-12801), the highest 5 pathways by unique sequences were $\mathrm{ABC}$ transporters,

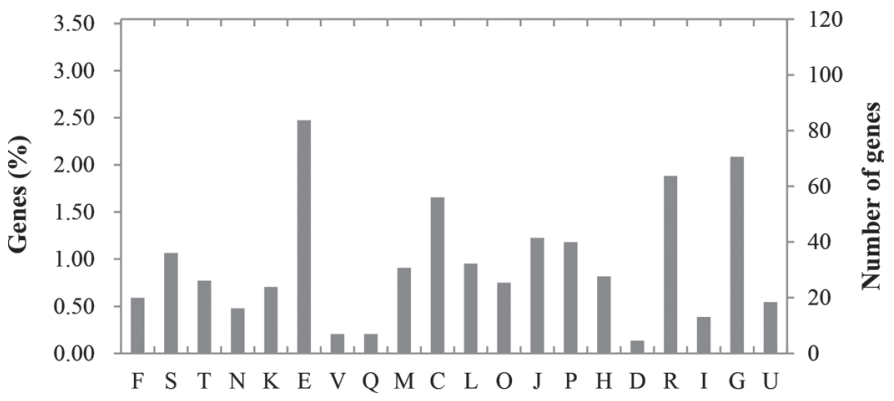

Figure 1. Clusters of orthologous group (COG) classification of transcripts of Cronobacter isolates. (F) Nucleotide transport and metabolism; (S) function unknown; (T) signal transduction mechanisms; (N) cell motility; (K) transcription; (E) amino acid transport and metabolism; (V) defense mechanisms; (Q) secondary metabolites biosynthesis, transport and catabolism; (M) cell wall/membrane/envelope biogenesis; (C) energy production and conversion; (L) replication, recombination, and repair; $(\mathrm{O})$ posttranslational modification, protein turnover, chaperones; $(\mathrm{J})$ translation, ribosomal structure, and biogenesis; (P) inorganic ion transport and metabolism; $(\mathrm{H})$ coenzyme transport and metabolism; (D) cell cycle control, cell division, chromosome partitioning; (R) general function prediction only; (I) lipid transport and metabolism; (G) carbohydrate transport and metabolism; (U) intracellular trafficking, secretion, and vesicular transport. 
2-component system (131), purine metabolism (77), pyrimidine metabolism (63), and ribosome (59).

\section{Mapping and Transcriptome Data}

The transcript reads were mapped to the complete genome of C. sakazakii ATCC BAA-894; we found that $82.35 \%$ (G362) and $80.38 \%$ (L3101) of reads were a unique match, and 1.58 and $4.11 \%$ of reads were multiple matches (Figure 3A). In addition, 16.07 and $15.51 \%$ of reads were not mapped to a complete genome of C. sakazakii ATCC BAA-894. The distribution of transcripts and their RPKM values were overviewed in Figure 3B and Figure 3C, respectively. From Figure $3 \mathrm{~B}$, the median numbers of RPKM values are less than 100 in G362 and L3101, and the maximum of RPKM is more than 500. In Figure 3C, the RPKM values of most of transcripts are more than 50, and RPKM values less than 3 are about 500 transcripts.

\section{Volcano Analysis of Differential Expression Genes}

To discover differential expression genes (DEG) between G362 and L3101, the gene expression levels were calculated using the RPKM method. Both $P$-value $<0.05$ and 1.0-fold change differences of $\log _{2}$ RPKM values between these 2 isolates were used as criteria to determine the significant DEG. The distribution of DEG is conducted using Volcano plots in Figure 4, and the expression changes of most of the DEG between G362 and L3101 are less than 2-fold.

\section{GO Enrichment and Heat Map Analysis of Typical DEG}

Among differentially expressed genes, typical DEG $(\mathrm{n}=96)$ were selected for heat map analysis (Figure 5A) and GO enrichment analysis (Figure 5B) and the predominant DEG include flagella-associated genes, outer membrane protein genes, and regulatory genes.
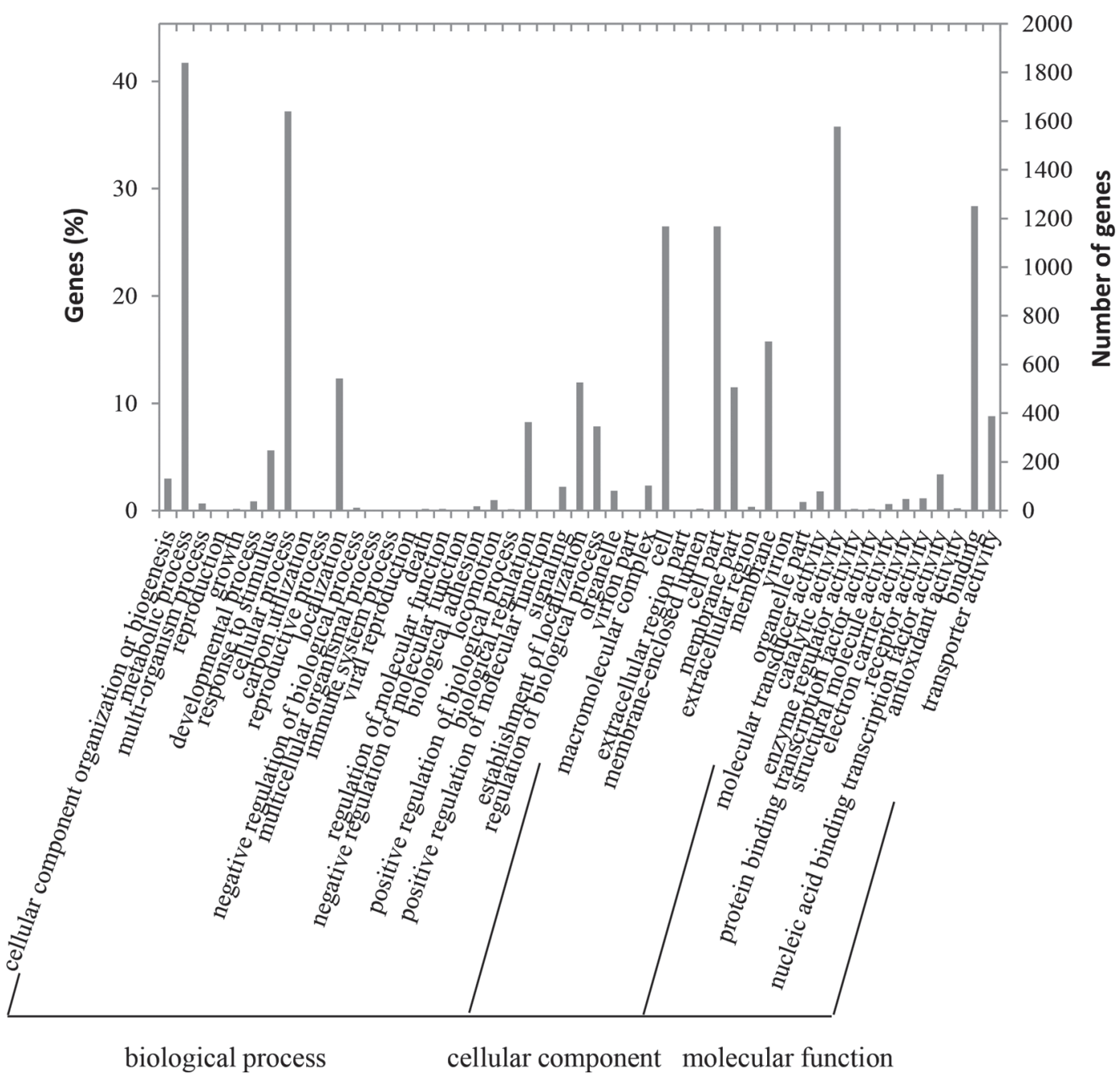

Figure 2. Gene ontology functional annotation of transcripts based on BLAST uniprot data. 


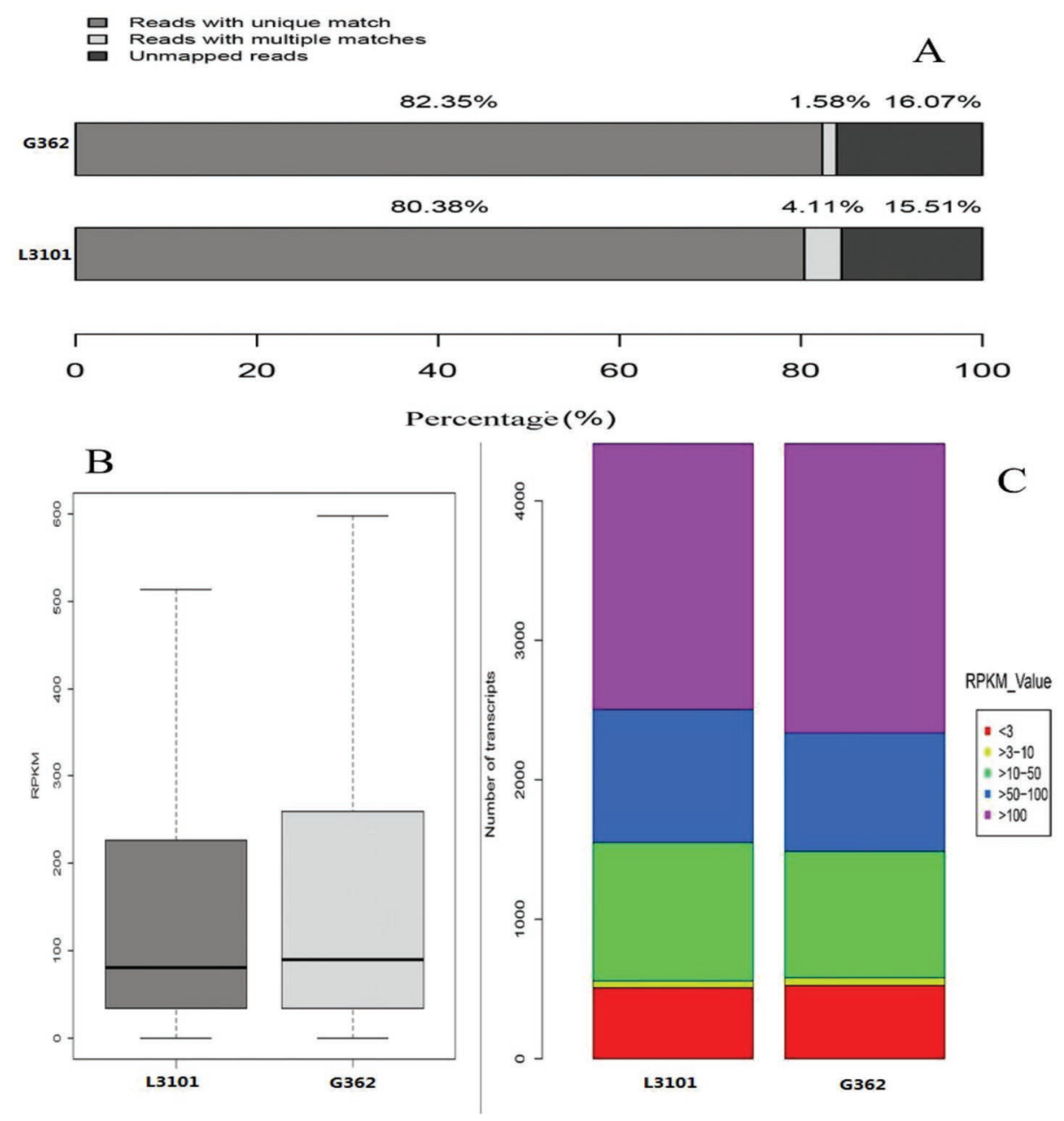

Figure 3. The alignment, distribution, and reads per kilobase of exon model per million mapped reads (RPKM) values of transcriptome of G362 and L3101. (A) Alignment of transcripts with reference database (genome of Cronobacter sakazakii ATCC BAA-894); (B) Box plot of RPKM value of transcripts of G362 and L3101 containing maximum, upper quartile (upper line of box), median (middle line), lower quartile (bottom line of box), minimum from top to bottom; (C) Distribution of RPKM values of G362 and L3101. Color version available online.

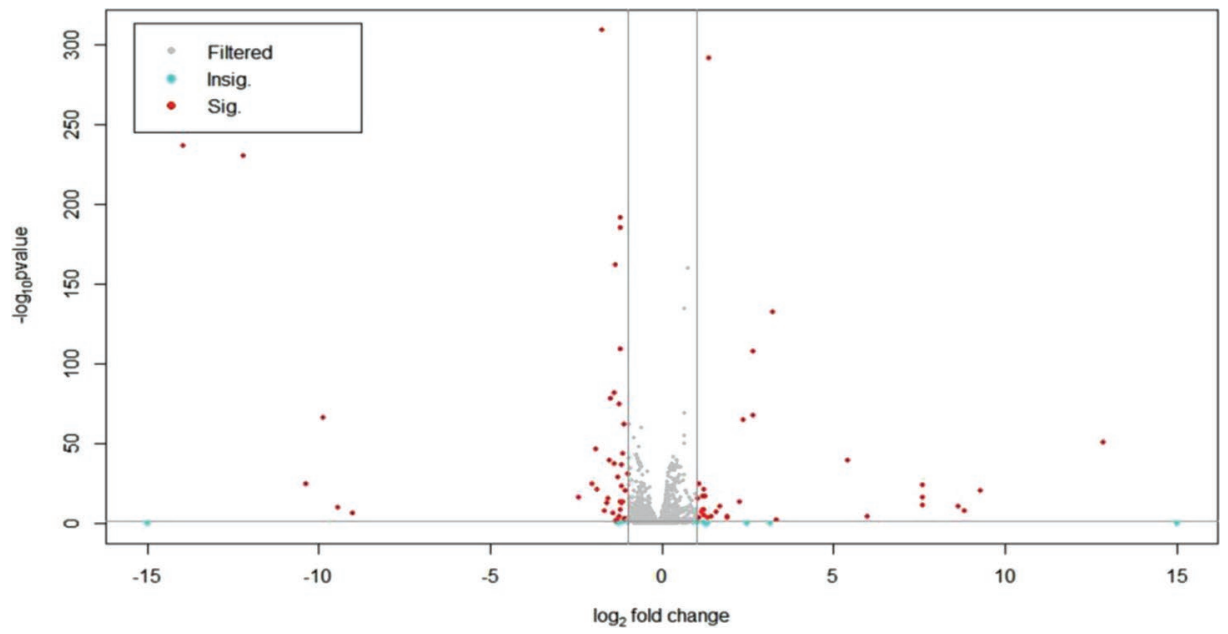

Figure 4. Volcano analysis of differential expression genes (DEG) between G362 and L3101. Both $P$-value $<0.05$ and 1.0 -fold change differences of $\log _{2}$ reads per kilobase of exon model per million mapped reads (RPKM) values between these 2 isolates were used as criteria to determine the significant DEG. Color version available online. 
In Table 2, major DEG with gene locus, expression products, and fold changes are listed. The flagella-related genes including $f l g B, f l g L, f l i T, f l i D, f l g N$, fliR, and flgK were significantly expressed between G362 and L3101. The expression abundance of chemotaxis genes including che $Y$ and che $R$ is significantly increased in G362. In addition, expression abundance of outer membrane protein and lipoprotein genes (omp $W$, slyB, blc, lolA, and tolC) was upregulated in G362. Regulation factors, including che $Y$, Bss, fliZ, flgM, and fliA genes, were also upregulated in G362, whereas sdiA and $f l h D$ were upregulated in L3101. Interestingly, the expression differences of potential virulence-related factors, such as hemolysin III (hlyIII) and hemolysin expression modulating protein $(h h a)$, are first observed between G362 and L3101. In addition, hlyIII and hha were upregulated and downregulated in G362, respectively. Furthermore, 3 hypothetical protein genes (ESA_01022, ESA_01609, and ESA_00609) were expressed only in G362, and expression abundance of 2 hypothetical protein genes (ESA_01625 and ESA_01779) were 9.40- and 12.65fold greater than those seen for L3101.

Furthermore, the relative expression of partial genes ( fliC, ompW, sdiA, flgM, and flhD) in Figure 6 was found to be consistent with the results by transcriptome analysis.

\section{DISCUSSION}

In this study, we have successfully identified potential virulence factors that were differentially expressed between a highly virulent strain and a lesser virulent strain using transcriptome analysis. In Table 2, DEG include flagella-associated genes, outer membranous protein, lipoprotein genes, and important regulators, which provided valuable information for the pathogenic mechanism of Cronobacter. Interestingly, we also found that the fold changes of most of genes between G362 and L3101 were less than 2. The relatively small differences may be explained that Cronobacter species are opportunistically foodborne pathogens and definite virulence factors are not revealed.

The $f l h D$ and $f l g M$ were reported to be the flagellar transcriptional activator and negative regulator, respectively, of flagellin synthesis (Soutourina and Bertin, 2003). After incubation in the TSB at $37^{\circ} \mathrm{C}$ for $16 \mathrm{~h}$, the flagella synthesis in G362 and L3101 were analyzed using a transmission electron microscope. In Supplemental Figure S2 (https://doi.org/10.3168/jds.2017 -12801), the significant changes of flagella synthesis were observed between G362 and L3101. The abundant flagella in $\mathrm{L} 3101$ at $37^{\circ} \mathrm{C}$ were synthesized compared with those in G362. Our findings further confirmed the functions of $f l g M$ and $f l h D$ on flagella biosynthesis in
Cronobacter. In Table 2, the main structural genes such as $f l i C$ were upregulated in L3101 using RNA-seq in this study, whereas most of flagella-associated genes, such as $f l i D$ and $f l g B$, decreased in G362. This phenomenon indicated that flagella synthesis was a complicated process in Cronobacter. Ye et al. (2015) reported that the biofilm-forming ability of G362 was higher than that of L3101, but relatively stronger swimming motility was observed in L3101. Our results also further found that the relationship among motility, flagella biosynthesis, and biofilm formation was also complex in Cronobacter. The flagellin monomers through toll-like receptor (TLR) 5 can trigger proinflammatory and adaptive immune responses of mammalian hosts (Ramos et al., 2004). So, sufficient flagellin in L3101 might contribute to the protective immune responses through TLR5 mechanism. It is inferred that the functions of flagella on the virulence of opportunistic pathogen-Cronobacter may depend on multiple factors such as species or strain, biofilm formation, and host immune system.

In other species, flagella contributed to the virulence of pathogenic bacteria through chemotaxis, adhesion to, and invasion of host cells (Ramos et al., 2004). For instance, flagella motility is required for colonization of intestinal mucosa in Vibrio cholerae (Krukonis and DiRita, 2003) and invasion of epithelial cells in Yersinia enterocolitica (Young et al., 2000). Flagella also contributed to the adherence of Clostridium difficile to mucus (Tasteyre et al., 2001). Intestinal invasion of Salmonella enterica serovar Typhimurium in the avian host is dose dependent and does not depend on motility and chemotaxis (Olsen et al., 2013). Legionella pneumophila flagellum can induce the protective immunities in an A/J mouse model (Ricci et al., 2005). The $f l h A$ and $\operatorname{mot} A$ were reported to play an important role in the cold tolerance of Listeria monocytogenes (Mattila et al., 2011). In Campylobacter jejuni, temperaturedependent FlgM/FliA complex formation regulated flagella length (Wösten et al., 2010). Furthermore, type III secretion systems in Salmonella serve as an additional mechanism for export and injection of virulence factors (Galán, 2001).

In Cronobacter, flagella from 5 Cronobacter species were involved in the inflammatory responses on TLR5 recognition and contributed to the pathogenicity of these Cronobacter species (Cruz-Córdova et al., 2012). Flagella biosynthesis is a complex process and flagella are related to motility, biofilm formation, and immune responses of hosts. So, the detailed functions and regulation of flagella on pathogenicity of Cronobacter isolates remain to be revealed.

Previous reports stated that outer membrane proteins, such as ompA and ompX, were identified to be involved in enhancing invasion and adhesion of $C$. saka- 
A

$\begin{array}{lll}-2.0 & 0.0 & 2.0\end{array}$

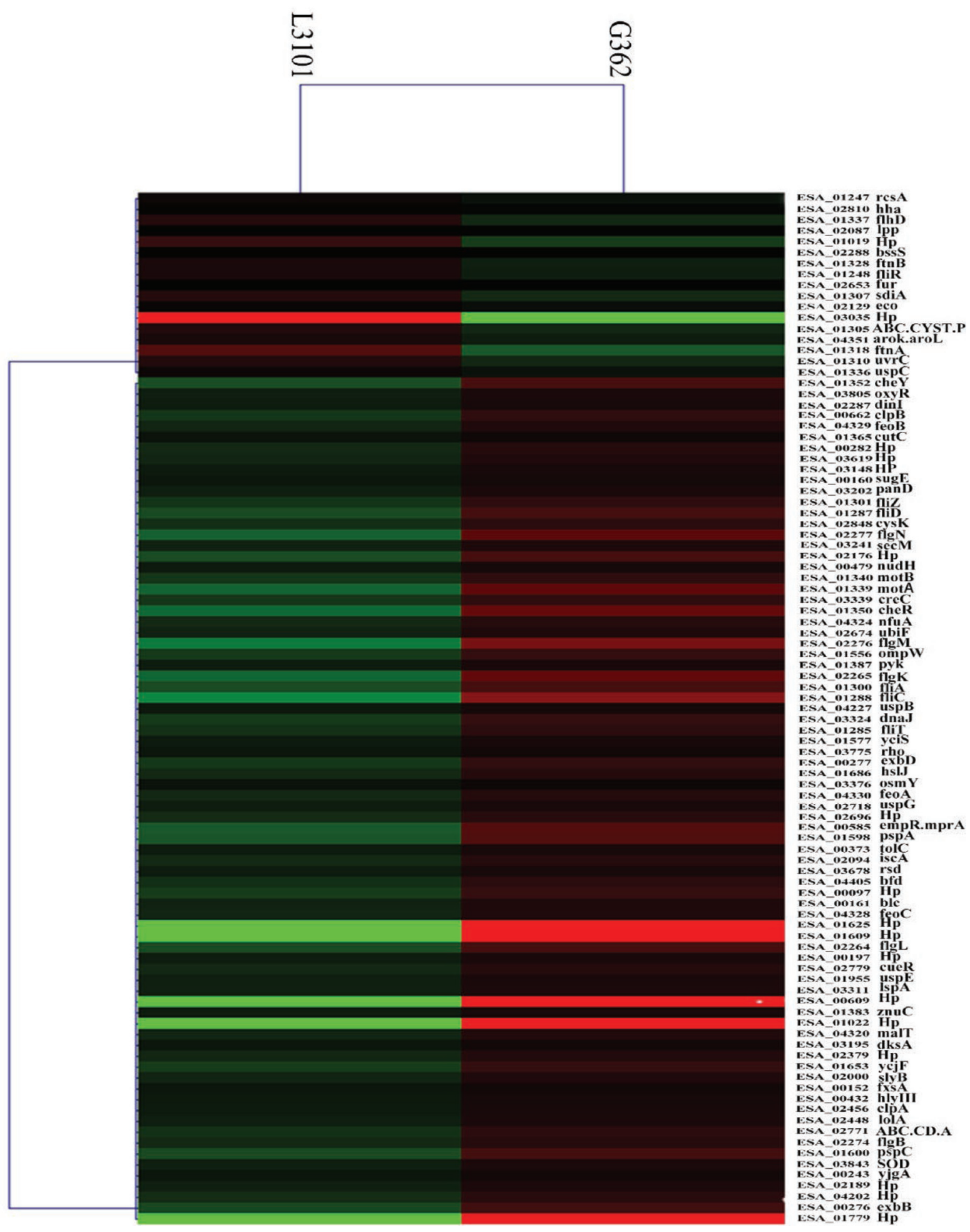

Figure 5. The heat map and gene ontology (GO) enrichment analysis of typically differential expression genes (DEG) between G362 and L3101. (A) Heat map analysis of DEG; (B) GO enrichment analysis of DEG. Color version available online. 


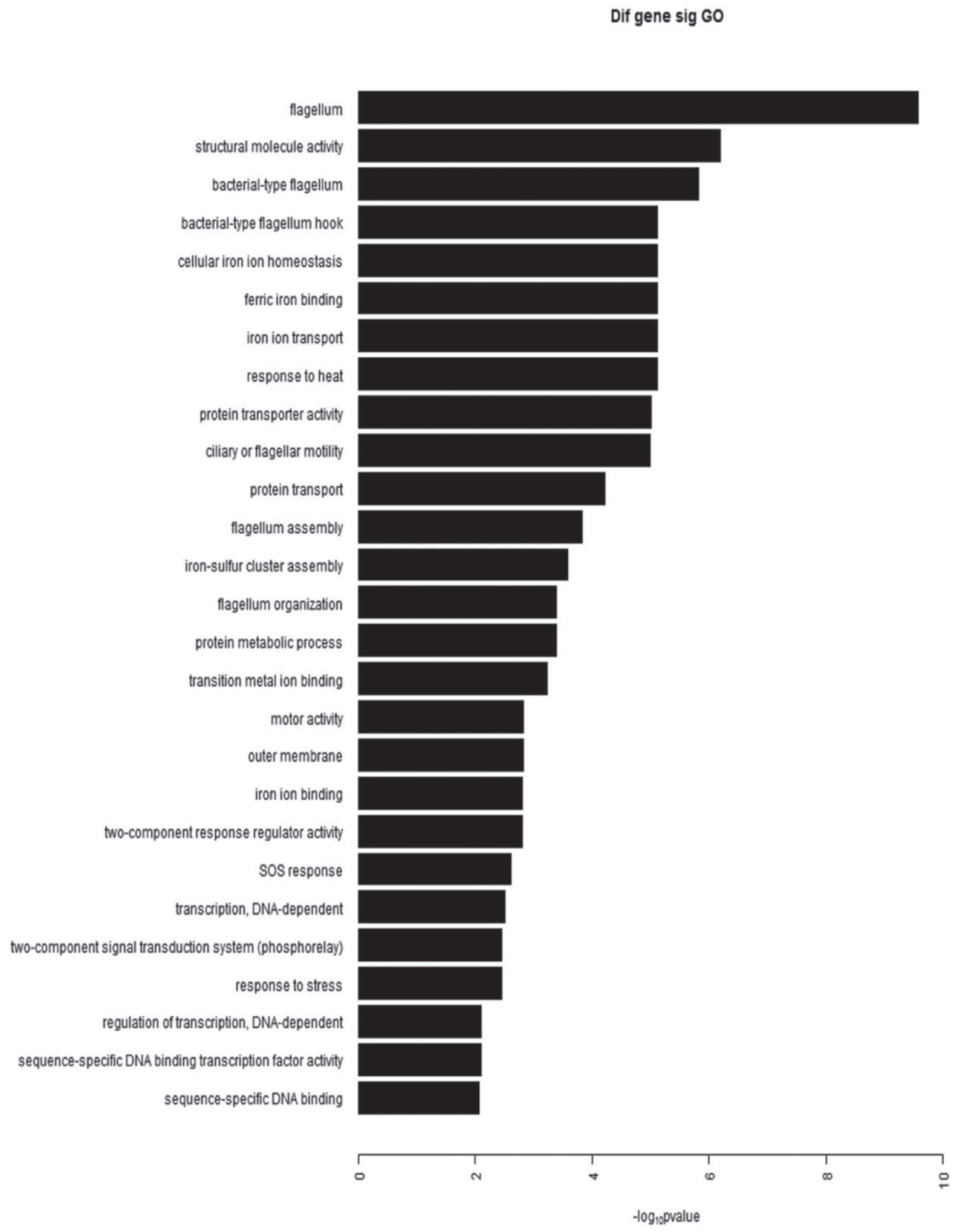

Figure 5 (Continued). The heat map and gene ontology (GO) enrichment analysis of typically differential expression genes (DEG) between G362 and L3101. (A) Heat map analysis of DEG; (B) GO enrichment analysis of DEG. Color version available online. 
Table 2. Typical differential expression genes (DEG) between G362 and L3101

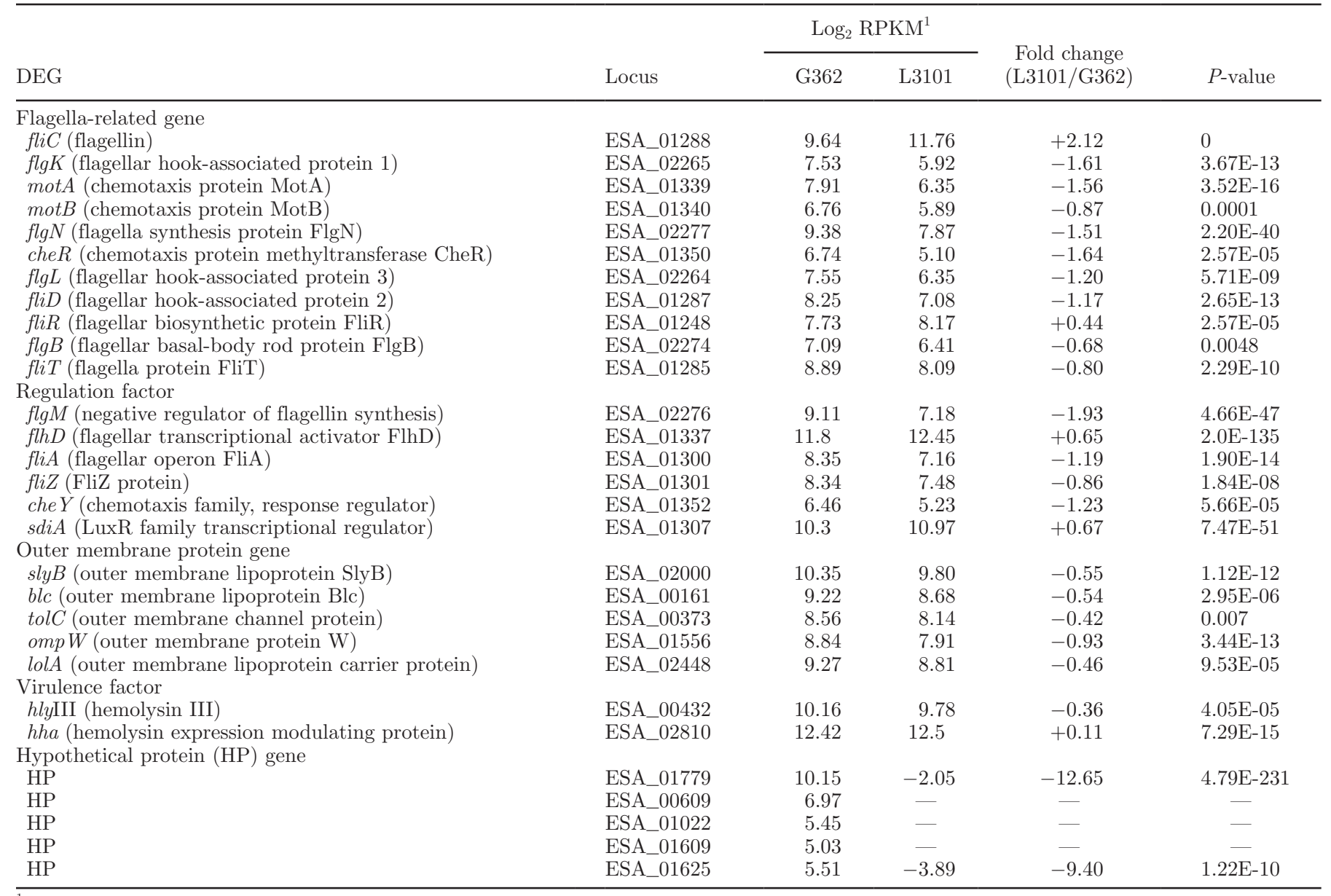

${ }^{1} \mathrm{RPKM}=$ reads per kilobase of exon model per million mapped reads.

zakii (Mohan Nair and Venkitanarayanan, 2007; Kim et al., 2010; Mittal et al., 2009). Using the 2-dimensional electrophoresis method, ompA, LuxS, and Dps proteins and membranous proteins (enzV, ompX, lptE, pstB, and $\mathrm{OsmY}$ ) were found to be potential factors involved in virulence of Cronobacter (Ye et al., 2015, 2016). Another study indicated that at least 11 of these proteins, such as fliC, ClpB, and $\mathrm{SrfC}$, may be involved in the pathogenesis of C. sakazakii (Du et al., 2015). In this study, potential outer membrane protein or lipoprotein genes such as omp $W$, slyB, blc, lolA, and tolC were first reported to be potentially related to virulence of Cronobacter at the mRNA level. Using whole-transcriptome sequencing to determine the gene abundance after interaction of C. sakazakii ATCC BAA-894 with HCT-8 cell, some flagella genes, virulence factors involved in adherence and outer membrane protein genes, were upregulated in the adherent C. sakazakii ATCC BAA894 strain on HCT-8 cells compared with the cultured bacteria in the cell-free medium (Jing et al., 2016). The omp $W$ is the virulence factor in non-O1 Vibrio chol- erae (Sharma and Chaturvedi, 2006) and is required for resistance to phagocytosis in Escherichia coli (Wu et al., 2013). The tolC gene was found to enhance the expression of the virulent factor (toxT) in Vibrio cholerae (Minato et al., 2011) and multidrug resistance and virulence in Francisella tularensis (Gil et al., 2006).

Interestingly, the expression levels of important regulators, such as sdiA, fliZ, and fliA, were also different between G362 and L3101. A chromosomal LuxR homolog encoded by sdiA was first found to be differentially expressed in virulent G362 and attenuated L3101 in this study. LuxR is a receptor of $N$-acyl-L-homoserine lactones (AHL) in quorum sensing (Walters and Sperandio, 2006). In E. coli O157, the expression of sdiA from high-copy-number plasmid reduced motility and the locus of enterocyte effacement-encoded genes, whereas the sdiA mutant contributed cell motility, curli gene expression, and biofilm formation (Kanamaru et al., 2000; Sharma et al., 2010). Ahmer (2004) reported that virulence was not affected by the sdiA gene in Salmonella. However, E. coli and Salmonella do not 


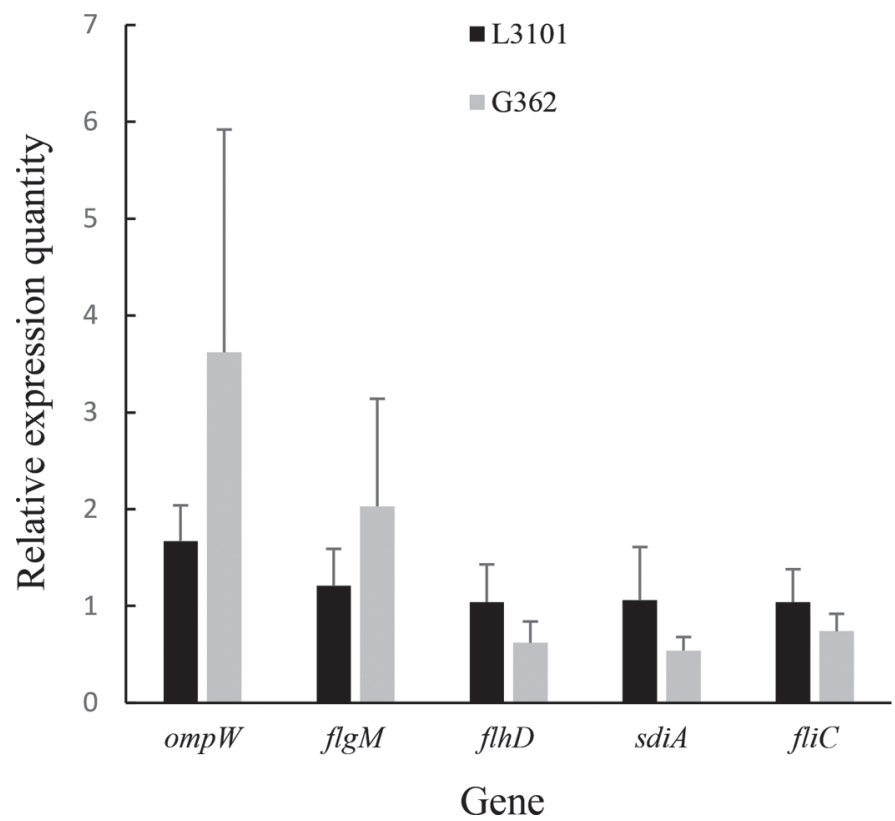

Figure 6. Relative expression quantity (mean $\pm \mathrm{SD}$ ) of partial genes between isolate G362 and L3101 by real-time PCR.

synthesize AHL, but can detect AHL for regulating the expression of genes. A subsequent study indicated that the binding of AHL to sdiA is essential to activate the sdiA regulator (Yao et al., 2006). Unlike E. coli and Salmonella, C. sakazakii can synthesize AHL (da Silva Araújo et al., 2012). The fliAZ operon plays a central role in Xenorhabdus nematophila, mediating the coordinate regulation of lipase activity and motility, and hemolysis and virulence (Lanois et al., 2008). In $S$. Typhimurium, fliZ mutation has been shown to significantly reduce hilA expression and intestinal colonization in mice (Lucas et al., 2000). In Salmonella enterica serovar Typhi, Eichelberg and Galán (2000) found that fliA disruption was reported to reduce significantly the expression of invasion genes clustered in the SPI-1-encoded type III secretion system.

Finally, expression abundance of hlyIII and $h h a$ was first revealed to be differentially expressed between G362 and L3101. Hemolysin-associated genes were involved in virulence or pathogenicity in E. coli (Bielaszewska et al., 2014), L. monocytogenes (Corr et al., 2006), and Vibrio (Lee et al., 2004; Wong et al., 2012). The roles of 2 factors on virulence or pathogenicity of Cronobacter was unknown.

In summary, the global molecular information at the mRNA level involved in virulence of Cronobacter using transcriptome analysis was successfully identified. The findings provided valuable knowledge for revealing the pathogenic mechanism of Cronobacter. These factors, such as flagella-associate genes, outer membrane pro- tein genes, and some regulators, may be related to motility, biofilm formation, and virulent characterization in Cronobacter through their expression and regulation. However, the detailed roles of these factors in pathogenicity of Cronobacter remain to be revealed.

\section{ACKNOWLEDGMENTS}

We gratefully acknowledge the financial support of the National Natural Science Foundation of China (31671951), the Anhui Provincial Grand Project Special of Science and Technology (15czz03109, China), the Science and Technology Planning Project of Guangdong Province (2016A050502033, China), Project of Science and Technology in Guangzhou (201604020036, China), and State Key Laboratory of Applied Microbiology Southern China Open Foundations, Guangzhou, China (SKLAM004-2015).

\section{REFERENCES}

Ahmer, B. M. 2004. Cell-to-cell signalling in Escherichia coli and Salmonella enterica. Mol. Microbiol. 52:933-945.

Audic, S., and B. A. Claverie. 1997. The significance of digital gene expression profiles. Genome Res. 7:986-995.

Baldwin, A., M. Loughlin, J. Caubilla-Barron, E. Kucerova, G. Manning, C. Dowson, and S. Forsythe. 2009. Multilocus sequence typing of Cronobacter sakazakii and Cronobacter malonaticus reveals stable clonal structures with clinical significance which do not correlate with biotypes. BMC Microbiol. 9:223.

Bielaszewska, M., T. Aldick, A. Bauwens, and H. Karch. 2014. Hemolysin of enterohemorrhagic Escherichia coli: Structure, transport, biological activity and putative role in virulence. Int. J. Med. Microbiol. 304:521-529.

CDC. January 13, 2012. CDC update: Investigation of Cronobacter infections among infants in the United States. Accessed Aug. 22, 2012. https://www.cdc.gov/cronobacter/investigation.html.

Chaudhuri, R. R., L. Yu, A. Kanji, T. T. Perkins, P. P. Gardner, J. Choudhary, D. J. Maskell, and A. J. Grant. 2011. Quantitative RNA-seq analysis of the Campylobacter jejuni transcriptome. Microbiology 157:2922-2932.

Choi, Y., K.-P. Kim, K. Kim, J. Choi, H. Shin, D.-H. Kang, and S. Ryu. 2012. Possible roles of LysR-type transcriptional regulator (LTTR) homolog as a global regulator in Cronobacter sakazakii ATCC 29544. Int. J. Med. Microbiol. 302:270-275.

Corr, S., C. Hill, and C. G. M. Gahan. 2006. An in vitro cell-culture model demonstrates internalin- and hemolysin-independent translocation of Listeria monocytogenes across M cells. Microb. Pathog. $41: 241-250$.

Cruz-Córdova,, A., L. M. Rocha-Ramirez, S. A. Ochoa, B. GonzálezPedrajo, N. Espinosa, C. Eslava, U. Hernandez-Chinas, G. Mendoza-Hernandez, A. Rodrıguez-Leviz, P. Valencia-Mayoral, S. Sadowinski-Pine, R. Hernandez-Castro, I. Estrada-Garcia, O. MunozHernandez, I. Rosas, and J. Xicohtencatl-Cortes. 2012. Flagella from five Cronobacter species induce pro-inflammatory cytokines in macrophage derivatives from human monocytes. PLoS One 7:e52091.

da Silva Araújo, F. D., L. M. R. Esper, A. Y. Kuaye, M. P. Sircili, and A. J. Marsaioli. 2012. N-Acyl-homoserine lactones from Enterobacter sakazakii (Cronobacter spp.) and their degradation by Bacillus cereus enzymes. J. Agric. Food Chem. 60:585-592.

Dong, C. S., G. P. Zhao, M. Zhong, Y. Yue, L. Wu, and S. D. Xiong. 2013. RNA sequencing and transcriptomal analysis of human monocyte to macrophage differentiation. Gene 519:279-287. 
Du, X.-J., R. Han, P. Li, and S. Wang. 2015. Comparative proteomic analysis of Cronobacter sakazakii isolates with different virulences. J. Proteomics 128:344-351.

Dugar, G., A. Herbig, K. U. Forstner, N. Heidrich, R. Reinhardt, K. Nieselt, and M. Cynthia. 2013. High-resolution transcriptome maps reveal strain-specific regulatory features of multiple Campylobacter jejuni isolates. PLoS Genet. 9:e1003495.

Eichelberg, K., and J. E. Galán.. 2000. The Flagellar Sigma Factor FliA $\left(\varsigma^{28}\right)$ regulates the expression of Salmonella genes associated with the centisome 63 type III secretion system. Infect. Immun. 68:2735-2743.

Emami, C. N., R. Mittal, L. Wang, H. R. Ford, and N. V. Prasadarao. 2012. Role of neutrophils and macrophages in the pathogenesis of necrotizing enterocolitis caused by Cronobacter sakazakii. J. Surg. Res. 172:18-28.

Feng, S., T. P. Eucker, M. K. Holly, M. E. Konkel, X. Lu, and S. Wang. 2014. Investigating the responses of Cronobacter sakazakii to garlic-drived organosulfur compounds: A systematic study of pathogenic-bacterium injury by use of high-throughput wholetranscriptome sequencing and confocal micro-raman spectroscopy. Appl. Environ. Microbiol. 80:959-971.

Food and Agriculture Organization of the United Nations/World Health Organization (FAO/WHO). 2008. Enterobacter sakazakii (Cronobacter spp.) in powdered follow-up formulae. Microbiological Risk Assessment Series No. 15. Food and Agriculture Organization of the United Nations/World Health Organization, Rome Italy.

Franco, A. A., L. Hu, C. J. Grim, G. Gopinath, V. Sathyamoorthy, K. G. Jarvis, C. Lee, J. Sadowski, J. Kim, M. H. Kothary, B. A. McCardell, and B. D. Tall. 2011. Characterization of putative virulence genes on the related RepFIB plasmids harbored by Cronobacter spp. Appl. Environ. Microbiol. 77:3255-3267.

Galán,, J. E. 2001. Salmonella interaction with host cells: Types III secretion at work. Annu. Rev. Cell Dev. Biol. 17:53-86.

Gil, H., G. J. Platz, C. A. Forestal, M. Monfett, C. S. Bakshi, T. J. Sellati, M. B. Furie, J. L. Benach, and D. G. Thanassi. 2006. Deletion of TolC orthologs in Francisella tularensis identifies roles in multidrug resistance and virulence. Proc. Natl. Acad. Sci. USA 103:12897-12902

Iversen, C., N. Mullane, B. McCardell, B. D. Tall, A. Lehner, S. Fanning, R. Stephan, and H. Joosten. 2008. Cronobacter gen. nov., a new genus to accommodate the biogroups of Enterobacter sakazakii, and proposal of Cronobacter sakazakii gen. nov., comb. nov. Cronobacter malonaticus sp. nov., Cronobacter turicensis sp. nov., Cronobacter muytjensii sp. nov., Cronobacter dublinensissp. nov., Cronobacter genomo-species 1, and of three subspecies, Cronobacter dublinensis ssp. dublinensis ssp. nov., Cronobacter dublinensis ssp. lausannensis ssp. nov. and Cronobacter dublinensis ssp. lactaridi ssp. nov. Int. J. Syst. Evol. Microbiol. 58:1442-1447.

Jing, C. E., X. Du, P. Li, and S. Wang. 2016. Transcriptome analysis of Cronobacter sakazakii ATCC BAA-894 after interaction with human intestinal epithelial cell line HCT-8. Appl. Microbiol. Biotechnol. 100:311-322.

Joseph, S., E. Cetinkaya, H. Drahovske, A. Levican, M. J. Figueras, and S. J. Forsythe. 2012. Cronobacter condimenti sp. nov., isolated from spiced meat and Cronobacter universalis sp. nov., a novel species designation for Cronobacter sp. genomospecies 1, recovered from a leg infection, water, and food ingredients. Int. J. Syst. Evol. Microbiol. 62:1277-1283.

Kanamaru, K., I. Tatsuno, T. Tobe, and C. Sasakawa. 2000. SdiA, an Escherichia coli homologue of quorum-sensing regulators, controls the expression of virulence factors in enterohaemorrhagic Escherichia coli O157: H7. Mol. Microbiol. 38:805-816.

Kim, K., K. P. Kim, J. Choi, J. A. Lim, J. Lee, S. Hwang, and S. Ryu. 2010. Outer membrane proteins A (OmpA) and X (OmpX) are essential for basolateral invasion of Cronobacter sakazakii. Appl. Environ. Microbiol. 76:5188-5198.

Kothary, M. H., B. A. McCardell, C. D. Frazar, D. Deer, and B. D. Tall. 2007. Characterization of the zinc-containing metalloprotease encoded by $z p x$ and development of a species-specific detec- tion method for Enterobacter sakazakii. Appl. Environ. Microbiol. $73: 4142-4151$.

Krukonis, E. S., and V. J. DiRita. 2003. From motility to virulence: Sensing and responding to environmental signals in Vibrio cholerae. Curr. Opin. Microbiol. 6:186-190.

Kucerova, E., S. W. Clifon, X. Q. Xia, F. Long, S. Porwollik, L. Fulton, C. Fronick, P. Minx, K. Kyung, W. Warren, R. Fulton, D. Feng, A. Wollam, N. Shah, V. Bhonagiri, W. E. Nash, K. Hallwworth-Pepin, R. K. Wilson, M. McClelland, and S. J. Forsythe. 2010. Genome sequence of Cronobacter sakazakii BAA-894 and comparative genomic hybridization analysis with other Cronobacter species. PLoS One 5:e9556.

Lanois, A., G. Jubelin, and A. Givaudan. 2008. FliZ, a flagellar regulator, is at the crossroads between motility, haemolysin expression and virulence in the insect pathogenic bacterium Xenorhabdus. Mol. Microbiol. 68:516-533.

Lee, S. E., P. Y. Ryu, S. Y. Kim, Y. R. Kim, J. T. Koh, O. J. Kim, S. S. Chung, H. E. Choy, and J. H. Rhee. 2004. Production of Vibrio vulnificus hemolysin in vivo and its pathogenic significance. Biochem. Biophys. Res. Commun. 324:86-91.

Lucas, R. L., C. P. Lostroh, C. C. DiRusso, M. P. Spector, B. L. Wanner, and C. A. Lee. 2000. Multiple factors independently regulate hilA and invasion gene expression in Salmonella enterica serovar typhimurium. J. Bacteriol. 182:1872-1882.

Mattila, M., M. Lindstrom, P. Somervuo, A. Markkula, and H. Korkeala. 2011. Role of flhA and motA in growth of Listeria monocytogenes at low temperatures. Int. J. Food Microbiol. 148:177-183.

Minato, Y., S. R. Fassio, and C. C. Hase. 2011. TolC affects virulence gene expression in Vibrio cholerae. J. Bacteriol. 193:5850-5852.

Mittal, R., Y. Wang, C. J. Hunter, I. Gonzalez-Gomez, and N. V. Prasadarao. 2009. Brain damage in newborn rat model of meningitis by Enterobacter sakazakii: A role for outer membrane protein A. Lab. Invest. 89:263-277.

Mohan Nair, M. K., and K. S. Venkitanarayanan. 2007. Role of bacterial OmpA and host cytoskeleton in the invasion of human intestinal epithelial cells by Enterobacter sakazakii. Pediatr. Res. 62:664-669.

Olsen, J. E., K. H. Hoegh-Andersen, J. T. Rosenkrantz, C. Schroll, J. Casadesus, S. Aabo, and J. P. Christensen. 2013. Intestinal invasion of Salmonella enterica serovar Typhimurium in the avian host is dose dependent and does not depend on motility and chemotaxis. Vet. Microbiol. 165:373-377.

Pagotto, F. J., M. Nazarowec-White, S. Bidawid, and J. M. Farber. 2003. Enterobacter sakazakii: Infectivity and enterotoxin production in vitro and in vivo. J. Food Prot. 66:370-375.

Perkins, T. T., R. A. Kingsley, M. C. Fookes, P. P. Gardner, K. D. James, L. Yu, S. A. Assefa, M. He, N. J. Croucher, D. J. Pickard, D. J. Maskell, J. Parkhill, J. Choudhary, N. R. Thmson, and G. Dougan. 2009. A strand-specific RNA-Seq analysis of the transcriptome of the typhoid bacillus Salmonella typhi. PLoS Genet. 5:e1000569.

Ramos, H. C., M. Rumbo, and J. C. Sirard. 2004. Bacterial flagellins: Mediators of pathogenicity and host immune responses in mucosa. Trends Microbiol. 12:509-517.

Ricci, M. L., A. Torosantucci, M. Scaturro, P. Chiani, L. Baldassarri, and M. C. Pastoris. 2005. Induction of protective immunity by Legionella pneumophila flagellum in an A/J mouse model. Vaccine 23:4811-4820.

Sharma, A., and A. N. Chaturvedi. 2006. Prevalence of virulence genes (ctxA, stn, OmpW and tcpA) among non-O1 Vibrio cholerae isolated from fresh water environment. Int. J. Hyg. Environ. Health 209:521-526.

Sharma, V. K., S. M. Bearson, and B. L. Bearson. 2010. Evaluation of the effects of sdiA, a luxR homologue, on adherence and motility of Escherichia coli O157: H7. Microbiology 156:1303-1312.

Soutourina, O. A., and P. N. Bertin. 2003. Regulation cascade of flagellar expression in Gram-negative bacteria. FEMS Microbiol. Rev. 27:505-523.

Stoop, B., A. Lehner, C. Iversen, S. Fanning, and R. Stephan. 2009. Development and evaluation of rpoB based PCR systems to dif- 
ferentiate the six proposed species within the genus Cronobacter. Int. J. Food Microbiol. 136:165-168.

't Hoen, P. A. C., Y. Ariyurek, H. H. Thygesen, E. Vreugdenhil, R. H. A. M. Vossen, R. X. de Menezes, J. M. Boer, G. J. van Ommen, and J. T. den Dunnen. 2008. Deep sequencing-based expression analysis shows major advances in robustness, resolution and interlab portability over five microarray platforms. Nucleic Acids Res. 36:e141.

Tasteyre, A., M. C. Barc, A. Colligono, H. Boureau, and T. Karjalainen. 2001. Role of FliC and FliD flagellar proteins of Clostridium difficile in adherence and gut colonization. Infect. Immun. 69:7937-7940.

Walters, M., and V. Sperandio. 2006. Quorum sensing in Escherichia coli and Salmonella. Int. J. Med. Microbiol. 296:125-131.

Wilhelm, B. T., S. Marguerat, S. Watt, F. Schubert, V. Wood, I. Goodhead, C. J. Penkett, J. Rogers, and J. Bahler. 2008. Dynamic repertoire of a eukaryotic transcriptome surveyed at single-nucleotide resolution. Nature 453:1239-1243.

Wong, S. K., X.-H. Zhang, and N. Y. S. Woo. 2012. Vibrio alginolyticus thermolabile hemolysin (TLH) induces apoptosis, membrane vesiculation and necrosis in sea bream erythrocytes. Aquaculture 330-333:29-36.

Wösten, M. M., L. van Dijk, A. K. J. Veenendaal, M. R. de Zoete, N. M. C. Bleumink-Pluijm, and J. P. M. van Putten. 2010. Temperature-dependent FlgM/FliA complex formation regulates Campylobacter jejuni flagella length. Mol. Microbiol. 75:1577-1591.
Wu, X.-B., L.-H. Tian, H.-J. Zou, C.-Y. Wang, Z.-Q. Yu, C.-H. Tang, F.-K. Zhao, and J. Y. Pan. 2013. Outer membrane protein OmpW of Escherichia coli is required for resistance to phagocytosis. Res. Microbiol. 164:848-855.

Xu, T., X. D. Guo, H. Wang, X. Y. Du, X. Y. Gao, and D. J. Liu. 2013. De novo transcriptome assembly and differential gene expression profling of three Caprahircus skin types during anagen of the hair growth cycle. Int. J. Genomics 2013:269191.

Yao, Y., M. A. Martinez-Yamout, T. J. Dickerson, A. P. Brogan, P. E. Wright, and H. J. Dyson. 2006. Structure of the Escherichia coli quorum sensing protein SdiA: Activation of the folding switch by acyl homoserine lactones. J. Mol. Biol. 355:262-273.

Ye, Y. W., R. Jiao, H. Li, Q. P. Wu, J. M. Zhang, and X. Zhong. 2015. The membrane proteins involved in virulence of Cronobacter sakazakii virulent G362 and attenuated L3101 isolates. Front. Microbiol. 6:1238.

Ye, Y. W., H. Li, N. Ling, Y. J. Han, Q. P. Wu, X. K. Xu, R. Jiao, and J. N. Gao. 2016. Identification of potential virulence factors of Cronobacter sakazakii isolates by comparative proteomic analysis. Int. J. Food Microbiol. 217:182-188.

Young, G. M., J. L. Badger, and V. L. Miller. 2000. Motility is required to initiate host cell invasion by Yersinia enterocolitica. Infect. Immun. 68:4323-4326. 